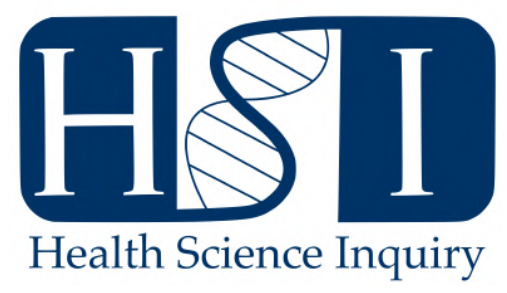

\title{
Leveraging 'omic' Technologies to Tailor Probiotic Treatment for Individuals
}

\author{
Emma Brownlie ${ }^{1}$ \\ ${ }^{1}$ Department of Molecular Genetics, University of Toronto
}

The World Health Organization defines probiotics as "live microorganisms which when administered in adequate amounts confer a health benefit on the host" [1]. The popularity of the beneficial effects of microorganisms has become progressively widespread over the decades, alongside our increasing awareness of the effects that bacteria can exert on health and disease such as inflammatory bowel disease (IBD) and non-alcoholic fatty liver disease (NAFLD) [2, 3]. By now, consumption of probiotics in an attempt to maintain health and prevent, ameliorate, or resolve disease has become routine. In the United States alone, recent estimates indicate that 3.9 million individuals regularly take probiotic supplements, and $60 \%$ of healthcare providers prescribe probiotics to patients [4]. This article will discuss probiotic treatment, current drawbacks, and how we can leverage big data approaches to increase precision and effectiveness of probiotics moving forward.

Given the prevalence of their use, it comes as no surprise that probiotics have become a highly popular field in academia and industry alike, ranging from basic to clinical research. Many studies have investigated the effects of probiotics, but the majority of those studies that have causally linked probiotics to positive outcomes have been carried out in animal models $[5,6]$. Several meta-analyses of studies performed in humans have indicated promising results, particularly in the context of ameliorating the symptoms of those people already suffering from diseases like IBD and NAFLD [2, 3]. However, these human studies tend to be less conclusive and in some cases yield contradictory results [7]. Additionally, risks associated with probiotic usage in certain populations such as those suffering from severe acute pancreatitis have been reported [8]. Clearly, although probiotics are a promising treatment option, there are many ways in which our understanding of the mechanism of action of these tools, as well as our application of them, can be improved.

To highlight this point, a recent study carried out by researchers at the Weizmann Institute has shown personspecific differences in the ability of probiotics to colonize the gut mucosa [9]. As expected, after treating healthy volunteers with probiotics these bacteria were found to be enriched in all participants. However, there was a highly individualized pattern of mucosal colonization by these pro- biotics, with some patients remaining stably colonized after treatment and others rapidly losing these bacteria. Although colonization of the gut may not be strictly necessary for bacteria to exert a therapeutic effect, it could still considerably affect local intestinal physiology, metabolism, and ecology. Critically, they also found that unique host and microbiome traits could predict whether or not colonization would occur - both the microbiome and the host transcriptome correlated with the 'permissiveness' or 'resistance' of the host to colonization with the probiotic strains. This emphasizes the extent to which individual characteristics can modify the effectiveness of a given treatment, and how this might significantly affect the outcome for an individual undergoing treatment.

These findings suggest the potential for a revolution in treatment from the undistinguished way in which probiotic treatments have been delivered to date. Precision medicine has become increasingly popular and accessible as we have developed ways to examine genomic, transcriptomic, proteomic, and metabolomics information - collectively referred to as 'omic' techniques. These approaches have already been implemented in fields such as immunology, oncology, and neurology to guide treatment regimens. For example, in oncology, transcriptomic information has been used extensively to classify tumours into subtypes and predict effectiveness of different therapies [10]. With research indicating that host response to probiotic treatment is highly individualized, it seems only logical to begin implementing these approaches in the administration of probiotics. This approach would allow researchers and clinicians to begin optimizing and tailoring treatment plans based on individual characteristics to optimize the success of treatments. Research studies would also allow the exploration of the mechanisms behind the beneficial effects of probiotics, and help distinguish cases where probiotics should not be used for health and safety reasons.

Currently, probiotics are a tool with incredible potential, but we need to begin leveraging the recent advances that we have seen in 'omic' technologies in order to improve treatment and gain a better understanding of how these bacteria mediate their effects. Examining the interaction between genes, environment, and microbiota is critical to understanding how probiotic treatments should be 
delivered and to whom. The implementation of 'omic' techniques would be a step towards turning probiotic usage into targeted treatment regimens. This would facilitate their use in a strategic manner to maximize effectiveness and minimize the potential pitfalls associated with treatment using probiotics.

\section{REFERENCES}

[1] Food and Agriculture Organization and World Health Organization Health Consultation. Health and nutritional properties of probiotics in food including powder milk with live lactic acid bacteria; 2001.

[2] Jia K, Tong X, Wang R, Song X. The clinical effects of probiotics for inflammatory bowel disease: A metaanalysis. Medicine. 2018;97(51).

[3] Loman BR, Hernández-Saavedra D, An R, Rector RS. Prebiotic and probiotic treatment of nonalcoholic fatty liver disease: a systematic review and meta-analysis. Nutrition reviews. 2018;76(11):822-839.

[4] Draper K, Ley C, Parsonnet J. Probiotic guidelines and physician practice: a cross-sectional survey and overview of the literature. Beneficial microbes. 2017;8(4):507-519.

[5] Raabis S, Li W, Cersosimo L. Effects and immune responses of probiotic treatment in ruminants. Veterinary immunology and immunopathology. 2019;.
[6] Yan F, Cao H, Cover TL, Washington MK, Shi Y, Liu $\mathrm{L}$, et al. Colon-specific delivery of a probiotic-derived soluble protein ameliorates intestinal inflammation in mice through an EGFR-dependent mechanism. The Journal of clinical investigation. 2011;121(6):22422253 .

[7] Rondanelli M, Faliva MA, Perna S, Giacosa A, Peroni G, Castellazzi AM. Using probiotics in clinical practice: Where are we now? A review of existing meta-analyses. Gut microbes. 2017;8(6):521-543.

[8] Besselink MG, van Santvoort HC, Buskens E, Boermeester MA, van Goor H, Timmerman HM, et al. Probiotic prophylaxis in predicted severe acute pancreatitis: a randomised, double-blind, placebo-controlled trial. The Lancet. 2008;371(9613):651-659.

[9] Zmora N, Zilberman-Schapira G, Suez J, Mor U, DoriBachash M, Bashiardes S, et al. Personalized gut mucosal colonization resistance to empiric probiotics is associated with unique host and microbiome features. Cell. 2018;174(6):1388-1405.

[10] Valdes Mora F, Handler K, Law AMK, Salomon R, Oakes SR, Ormandy CJ, et al. Single-cell transcriptomics in cancer immunobiology: the future of precision oncology. Frontiers in Immunology. 2018;9:2582.

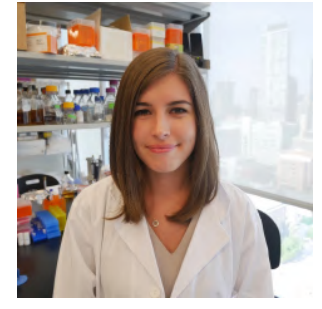

Emma is an MSc candidate at the University of Toronto in the department of Molecular Genetics. While completing a BScH at Queen's University, she spent summers working at the biotechnology firm DNA Genotek, where she initially became interested in studying the microbiota. Her current research focuses on leveraging in vitro approaches to examine bacterial interactions within the gut microbiota. 